\section{Thoracic endometriosis: rare presentation as a solitary pulmonary nodule with eccentric cavitations}

A 41-year-old woman who suffered from monthly haemoptysis for $>16$ years was referred to a chest surgeon due to an abnormal chest radiograph. The haemoptysis usually occurred on the first day of her menses and lasted $\sim 3$ days. She had poliomyelitis during her childhood and underwent two caesarean sections about two decades previously. There was no prior history of dysmenorrhoeal or abnormal vaginal bleeding.

Physical examinations and laboratory investigations were unremarkable. The chest radiograph (fig 1A) showed a right lower lobe nodule. CT scan (fig 1B,C) performed during her menstrual period demonstrated a well-demarcated subpleural ovoid tumour with eccentric cavitations and surrounding ground-glass opacities. Using video-assisted thoracoscopic surgery, a $2 \times 2 \times 2 \mathrm{~cm}$ nodule without pleural retraction was noticed (fig 2A). Wedge resection was performed smoothly. She did not receive hormonal therapy and remained uneventful in the subsequent 2-year follow-up. Microscopically, the

\section{Learning point}

- Thoracic endometriosis may present, though rarely, as a welldemarcated solitary pulmonary nodule and should be considered in a woman with long-term haemoptysis.

tumour was composed of abundant endometrial glands and stroma with focal fresh haemorrhage (fig 2B).

Thoracic endometriosis may involve the trachea, bronchi, lung parenchyma, pleura or the diaphragm. Pleural and diaphragmatic endometriosis usually causes chest pain and dyspnoea, and may be associated with pneumothorax, pleural effusion or haemothorax, whereas the tracheobronchial and parenchymal disease may present with periodic haemoptysis simultaneous with their menses. ${ }^{1}$ It is unclear whether hormonal therapy needs to be maintained after a complete resection of isolated intrapulmonary endometriosis since a controlled study is unavailable.

The radiological findings for catamenial haemoptysis are often normal, but they can show solitary or multiple pulmonary haziness displaying cyclic changes in $\operatorname{size}^{2}$ as a presentation of the menstrual haemorrhage in the adjacent alveolar spaces. For the present report, the unusually long duration of the history
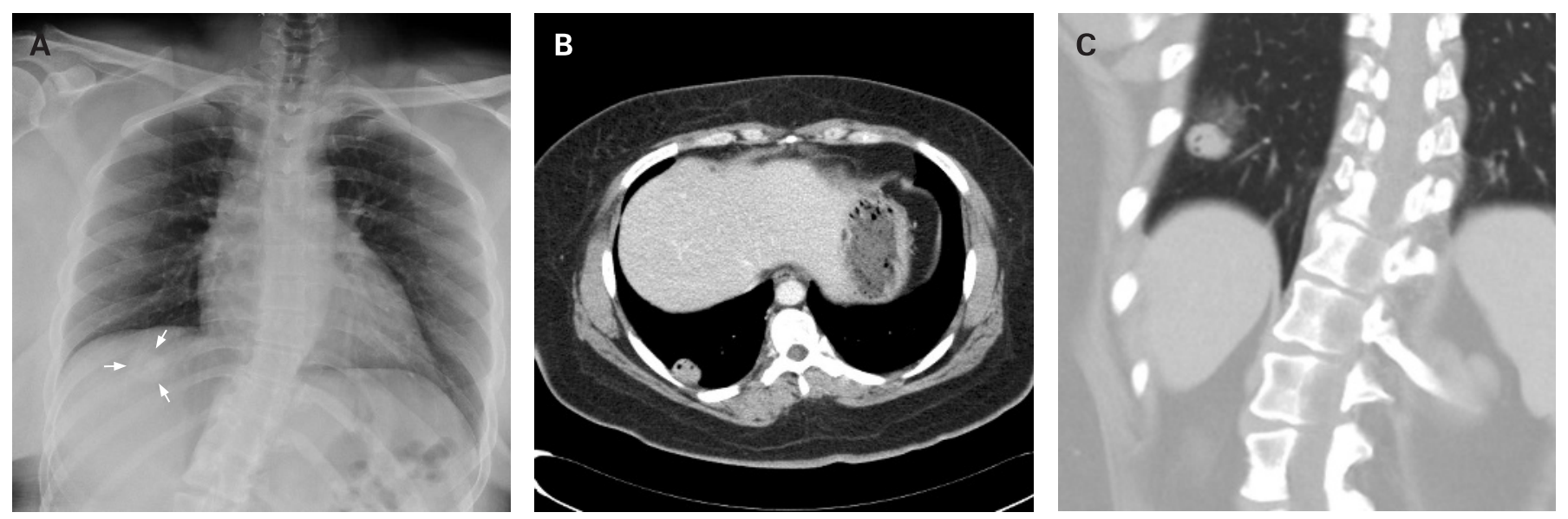

Figure 1 (A) Chest radiograph shows a solitary nodule (arrows) at the right lower lobe superimposed on the liver shadow. (B) CT scan of the chest demonstrated a well-demarcated subpleural ovoid tumour with eccentric cavitations at the right lower lobe. (C) Lung window of a reformatted coronal plane image reveals ground-glass opacities at the periphery of the tumour.
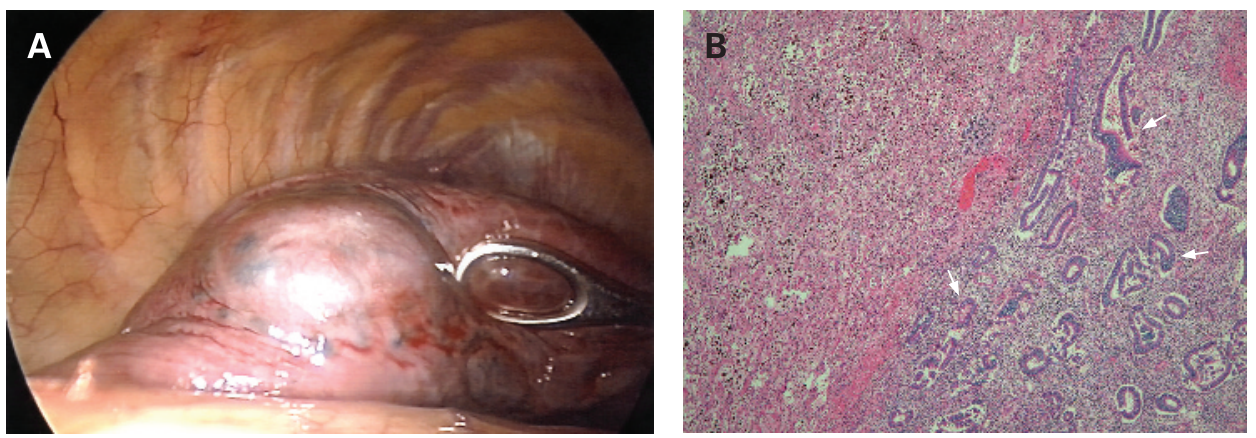

Figure 2 (A) A $2 \times 2 \times 2 \mathrm{~cm}$ nodule without pleural retraction was noticed during video-assisted thoracoscopic surgery. (B) Microscopic examination of the lung nodule revealed abundant ectopic endometrial glands (arrows) with stroma. The adjacent lung parenchyma showed many haemosiderinladen macrophages in the alveolar spaces $(\mathrm{H \& E}, \times 200)$. 
might be the reason for the endometrial tissue having developed such a sizeable solid nodule that is radiologically discernible.

\section{C-H Lee, ${ }^{1}$ Y-C Huang, ${ }^{1}$ S-F Huang, ${ }^{2}$ Y-K Wu, ${ }^{1}$ K-T Kuo ${ }^{3}$}

${ }^{1}$ Department of Internal Medicine, Buddhist Tzu Chi General Hospital, Taipei Branch, Taiwan; ${ }^{2}$ Department of Pathology, Buddhist Tzu Chi General Hospital, Taipei Branch, Taiwan; ${ }^{3}$ Department of Surgery, Buddhist Tzu Chi General Hospital, Taipei Branch, Taiwan

Correspondence to: Dr K-T Kuo, Department of Surgery, Buddhist Tzu Chi General Hospital, Taipei Branch, \#289 JianGuo Road, Xiandian City, Taipei County, 23142, Taiwan; doc2738h@gmail.com
Competing interests: None.

Patient consent: Obtained.

Thorax 2009;64:919-920. doi:10.1136/thx.2008.111294

\section{REFERENCES}

1. Joseph J, Sahn SA. Thoracic endometriosis syndrome: new observations from an analysis of 110 cases. Am J Med 1996;100:164-70.

2. Chung SY, Kim SJ, Kim TH, et al. Computed tomography findings of pathologically confirmed pulmonary parenchymal endometriosis. J Comput Assist Tomogr 2005;29:815-8.

\section{Pulmonary puzzle}

\section{ANSWER}

From the question on page 862

Broad-range bacterial PCR analysis was performed on the cerebral biopsy specimen ${ }^{1}$ and $16 \mathrm{~S}$ rDNA of Prevotella spp was detected. A diagnosis of chronic Prevotella spp infection was made. Prevotella spp are a group of Gram-negative anaerobes that colonise normal human mucosae and are recognised causes of oral, head and neck, and respiratory infection. Signs of chronic suppurative infection are typically present. Deep-seated pure Prevotella infections-notably CNS abscesses-may occur, probably as a result of haematogenous spread. ${ }^{2}$ Infections ensue when host factors (such as malnutrition and self-neglect) disrupt the normal commensal relationship. ${ }^{2}$ Chronic poor oral hygiene, alcoholism and a consequent risk of inhalation therefore put our patient at high risk of anaerobic pleuropulmonary infection with ensuing haematogenous dissemination.

This case illustrates the potential role of molecular identification techniques in diagnosing unusual causes of respiratory infection. Despite culture of blood, sputum, bronchoalveolar lavage fluid and brain tissue, no pathogen was isolated. The difficulty in isolating and culturing anaerobic organisms is well recognised, with organism survival and recovery rate adversely affected by specimen handling. ${ }^{3}$ These infections are frequently polymicrobial and generally are diagnosed based on clinical presentation. RT-PCR is a rapid and highly sensitive diagnostic tool that has been used to identify a wider array of pathogens than is achieved with concomitant culture, and also enables identification of pathogens present in numbers below the lower detection limit of traditional culture. ${ }^{45}$

The patient was treated with metronidazole for 6 weeks and his respiratory symptoms and confusion resolved, although he has persistent lower limb paresis.

Acknowledgements: The authors thank Dr John Hartley, Great Ormond Street Hospital, London who performed the PCR analysis. Thorax 2009;64:920. doi:10.1136/thx.2009.116293a

\section{REFERENCES}

1. Harris KA, Hartley JC. Development of broad-range $16 \mathrm{~S}$ rDNA PCR for use in the routine diagnostic clinical microbiology service. J Med Microbiol 2003;52:685-91.

2. Bartlett JG. The role of anaerobic bacteria in lung abscess. Clin Infect Dis 2005;40:923-5.

3. Winn WC Jr, Allen S, Janda WM, et al. The anaerobic bacteria. In: Winn WC Jr, Allen S, Janda WM, et al, eds. Koneman's color atlas and textbook of diagnostic microbiology. Philadelphia: Lippincott Williams and Wilkins, 2006:877-944.

4. Riggio MP, Aga H, Murray CA, et al. Identification of bacteria associated with spreading odontogenic infections by $16 \mathrm{~S}$ rRNA gene sequencing. Oral Surg Oral Med Oral Pathol Oral Radiol Endod 2007;103:610-7.

5. Boutaga K, van Winkelhoff AJ, Vandenbroucke-Grauls CM, et al. The additional value of real-time PCR in the quantitative detection of periodontal pathogens. J Clin Periodontol 2006;33:427-33. 